\title{
Mediastinoscopic Upper Mediastinal Lymph Node Dissection for Cervical Esophageal Carcinoma Using the LeftCervicalApproachUnder Pneumomediastinumin Thiel-Embalmed Cadavers
}

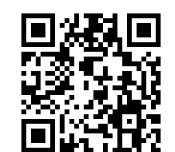

\author{
Yutaka Tokairin $^{1 *}$, Yasuaki Nakajima ${ }^{1}$, Kenro Kawada ${ }^{1}$, Akihiro Hoshino ${ }^{1}$, Takuya Okada $^{1}$, Tairo Ryotokuji ${ }^{1}$, Yuichiro \\ Kume $^{1}$, Yudai Kawamura ${ }^{1}$, Kazuya Yamaguchi ${ }^{1}$, Kagami Nagai ${ }^{1}$, Keiichi Akita ${ }^{2}$ and Yusuke Kinugasa ${ }^{1}$
}

${ }^{1}$ Department of Gastrointestinal Surgery, Tokyo Medical and Dental University, Japan

${ }^{2}$ Department of Clinical Anatomy, Tokyo Medical and Dental University, Japan

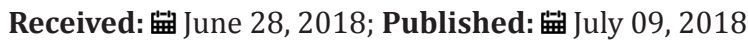

*Corresponding author: Yutaka Tokairin, Department of Gastrointestinal Surgery, Tokyo Medical and Dental University, 1-5-45 Yushima, Bunkyo-ku, Tokyo 113-8510, Japan

\begin{abstract}
Background:It is often difficult to perform complete upper mediastinal dissection for cervical esophageal cancer in the normal cervical surgery view. If a method to perform upper mediastinal dissection, including the left recurrent nerve lymph nodes (106recL), can be developed, then it may be possible to preform minimal invasive surgery without sternal splitting incisions or esophagectomy under right thoracotomy. We herein describe a new method for performing complete dissection of the upper mediastinal lymph nodes for cervical esophageal cancer under the pneumomediastinum using cadavers.
\end{abstract}

Methods:We developed a method for performing complete meidiastinoscopic upper mediastinal lymph node dissection for cervical esophageal carcinomain six Thiel-embalmed cadavers.

Procedures:A left cervical collar incision was made and open surgery on the left side was subsequently changed to the Pneumomediastinumin method after identifying the left recurrent nerve. The Alexis wound retractor was placed and deployed, and a single-port laparoscopic access device was then attached. The Pneumomediastinumin was then established and the 106recL lymph nodes were dissected along the left common carotid artery, subclavian artery, thoracic duct and the left recurrent nerve. The esophageal wall was divided from the membranous trachea. The Pneumomediastinumin can create a good view and lead to complete dissection of the upper mediastinum lymph nodes.

Discussion:We herein demonstrated that the upper mediastinal lymph node can be dissected usinglefttranscervical approach under Pneumomediastinumin and named this method "transcervicalmediastinoscopicupper-mediastinallymphnode dissection" (TCM-UMLD).TCM-UMLD is therefore considered to be a useful modality based on our experience with Thiel-embalmed human cadavers.

Keywords: Cervical Esophageal Cancer; Lymph Node Dissection; Mediastinal Lymph Node Dissection; Mediastinoscopy; Pneumomediastinum.

\section{Introduction}

It is often difficult to perform complete upper mediastinal dissection for cervical esophageal cancer in the normal cervical surgery view. If it is necessary to perform upper mediastinal dissection, including the left recurrent nerve lymph nodes (106recL), then a sternal splitting incision or esophagectomy under right thoracotomy must be performed. However, until recently, it has been difficult to diagnose accurately upper mediastinal lymph node metastasis. If lymph node metastasis is falsely positive, then dissection of the upper mediastinal lymph node by the sternal splitting incision or esophagectomy under right thoracotomy would be an unnecessary invasion. Therefore, if a method to perform upper mediastinal dissection, including the left recurrent nerve lymph nodes (106recL), can be developed, then it may be possible to perform minimal invasive surgery without sternal splitting incisions or esophagectomy under right thoracotomy. We herein describe a new method for performing complete dissection of the upper mediastinal lymph nodes for cervical esophageal cancer under the Pneumomediastinumin using cadavers,referred to as "transcervicalmediastinoscopicupper-mediastinallymphnode dissection (TCM-UMLD)".

\section{Material and Methods}

We developed a method for performing uppermediastinallymphnode dissection via the transcervical approach in 
six Thiel-embalmed human cadavers. The cadavers were donated to the Department of Anatomy, Tokyo Medical and Dental University. Before the patients died, they signed documents agreeing to donate their body for use in clinical studies. The format of the document meets the criteria for the Japanese "Act on Body Donation for Medical and Dental Education" guidelines.All cadavers were embalmed using the method previously described by Thiel [1,2]. The cadavers were embalmed in a water-based solution consisting of salt with a small amount of formaldehyde for fixation, boric acid for disinfection, glycol, chlorocresol and ethanol; this precipitation results in tissue homogenization. The skin is life-like, and the joints are fully flexible. We chiefly applied this method for upper mediastinal dissection. The procedures were performed using a pneumoperitoneum apparatus system (EVIS LUCERA SPECTRUM WM-NP1; OLYMPUS, Tokyo).In addition, the thoracic and abdominal aortas were inflated by the agar gel injection method in order to imitate live patient conditions.

\section{Procedure}

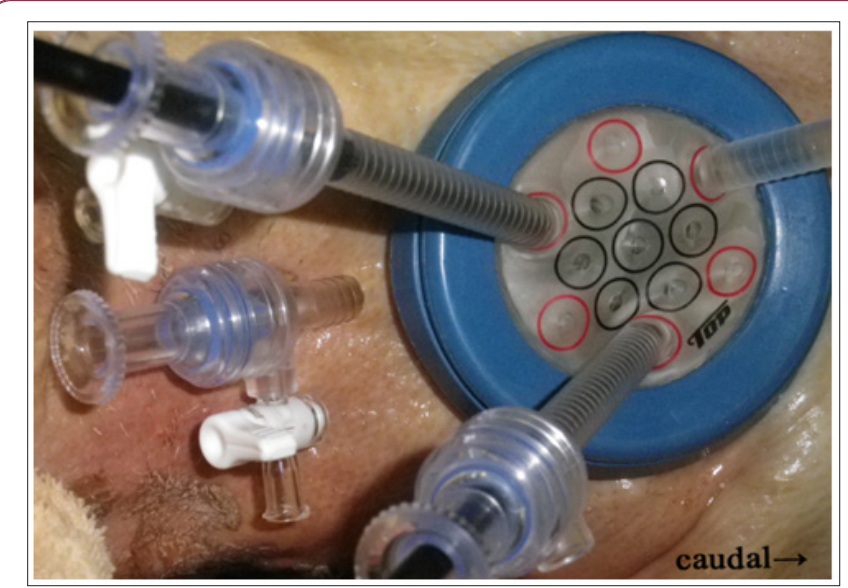

Figure 1: The layout of a single-port laparoscopic access device (SILS) and trocarsin a cadaver. The Alexis wound retractor is placed and deployed, a single-port laparoscopic access device (Free AccessTM;Top Corporation, Tokyo, Japan) is attachedand three $5 \mathrm{~mm}$ trocars are placed in a triangle configuration. The port for mediastinoscopy is inserted from a more cranial position.

A left cervical collar incision was made and a sufficient working space was made between the tracheoesophageal and the left carotid sheath. Open surgery on the left side was subsequently changed to the Pneumomediastinumin method after identifying the left recurrent nerve. The Alexis wound retractor (Applied Medical, Rancho Santa Margarita, CA, USA) was placed at the aforementioned space and deployed, a single-port laparoscopic access device (Free AccessTM; Top Corporation, Tokyo, Japan) was then attached and three $5 \mathrm{~mm}$ trocars were placed in a triangle configuration (Figure1). A Pneumomediastinuminwas then established with $\mathrm{CO}_{2}$ to a pressure of $10 \mathrm{mmHg}$, and the left recurrent nerve lymph nodes (106recL) were readily dissected along the left common carotid artery, subclavian artery, thoracic duct and dorsal area of the left recurrent nerve.The left recurrent nerve was observed around the aorta arch. The ramouscardiac us of the sympathetic nerve was clearly observed along the left subclavian artery in a different layer from which the left recurrent nerve was observed (Figure 2). In the Department of Clinical Anatomy of our institute, the ramous of the left recurrent nerve of the trachea was demonstrated previously (Figure 3). We also clearly demonstrated the ramous of the left recurrent nerve of the trachea in the cadavers under the pneumomediastinumin(Figure 4). Then, the esophageal wall was divided from the membranous trachea and the 106recL lymph node, the caudal area of the left recurrent nerve, was dissected. After these operations, I confirm how many lymph nodes dissected in 106recL area by thoracotomy.It is revealed that these lymph nodes are almost completely dissected in TCM-UMLD as well as in right thoracotomy.

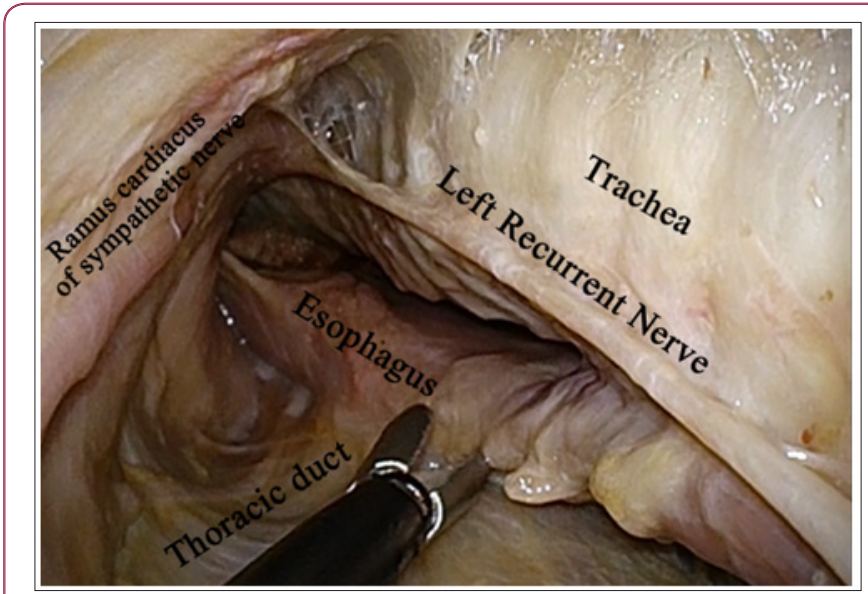

Figure 2: Left cervical upper mediastinal lymph nodes dissection under the pneumomediastinumin a cadaver. Pneumodissection separates loose connective tissue and enables us to dissect the left and posterior side of the esophagus along the left subclavian artery and thoracic duct. The left recurrent nerve is revealed to run at a different layer from the ramus cardiacus of the sympathetic nerve.

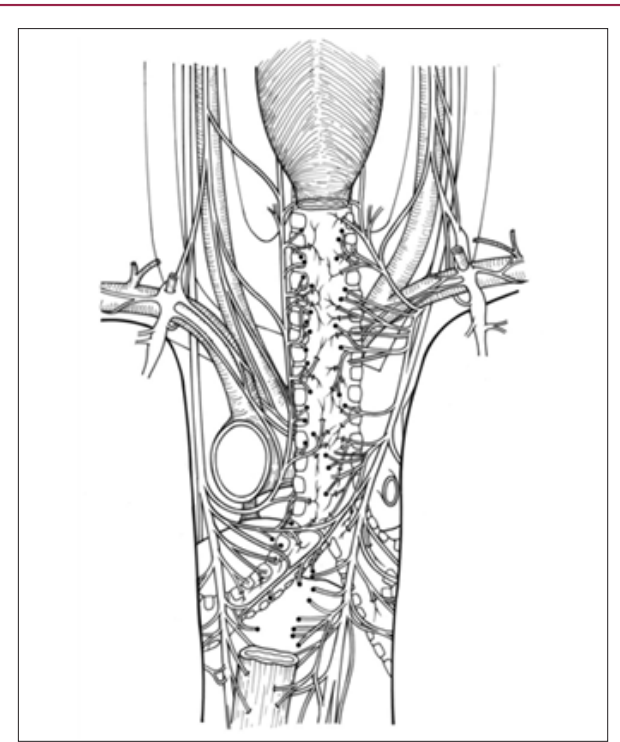

Figure 3: The schema of innervation for the trachea (autopsy).The innervation for the trachea is indicated. Distribution of the nerve for the trachea is observed from thevagus nerve in the right side and from the left recurrent nerve in the left side. 


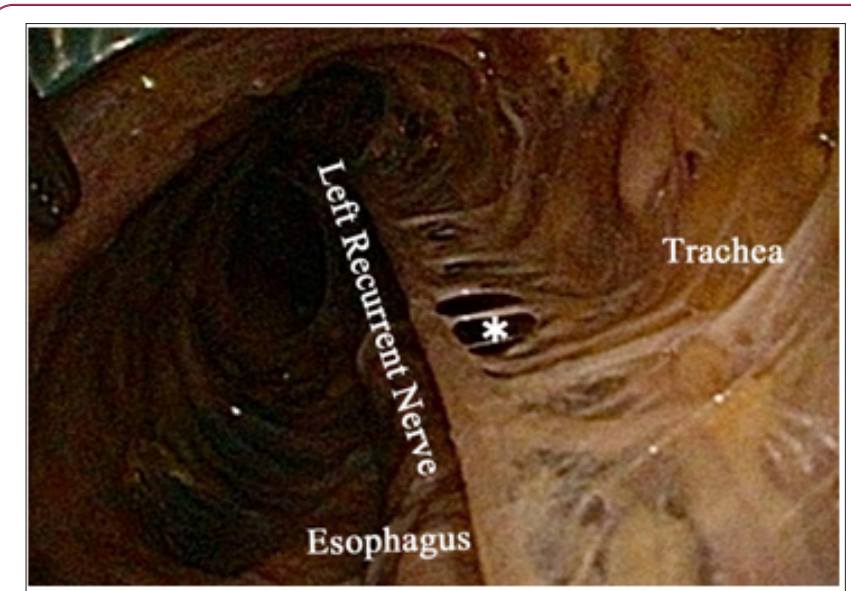

Figure 4: The innervation for the trachea in the left side of a cadaver.The nerve branch for the trachea $\left(^{*}\right)$ from the left recurrent nerve is observed as the ladder.

\section{Discussion}

Not many cases of cervical esophageal cancer have been observed and few reports have described [3-12].For the treatment of esophageal cancer, chemoradiation therapy and surgery was selected according to the guideline for esophageal cancer in Japan. Many problems are observed during cervical esophagectomy; for example, preservation of the laryngeal function, the proper approach method for upper mediastinal lymph node dissection and the range of lymph nodes dissection. Cervical esophageal cancer that cannot be radically dissected is typically recommended for upper mediastinal lymph node dissection. Only the lymph nodes that can be reached via the transcervical approach are dissected during cervical esophageal cancer. However, sometimes the lymph nodes cannot be completely dissected using the transcervical approach, and sternal splitting incisions or esophagectomy under right thoracotomy are needed for radical resection.We considered performing the 106recR lymph node dissection via the right transcervical approach during open surgery. It is difficult to dissect the 106recL lymph nodes via the left transcervical approach in open surgery. Thus, we determined that it is very important to dissect the 106recL lymph node via the left transcervical approach in the pneumomediastinum method.We demonstrated the use of pneumomediastinumesophagectomya bilateral transcervical and transhiatal approach under the pneumomediastinum and named this method "mediastinoscopicesophagectomy with lymph node dissection" (MELD) in cadavers[13].Our results indicated that the dissection of the upper mediastinal lymph node via the left transcervical approach is very effective for the effect of pneumodissection fed carbon dioxide gas. This pneumodissection separates loose connective tissue bloodlessly and enables us to expose rapidly the trachea, left subclavian artery, thoracic duct and left recurrent nerve.

Therefore, we believe that this method is suitable for upper mediastinal lymph dissection for cervical esophageal cancer. In addition, the augmentation effect by mediastinoscopic surgery enabled us to dissect sufficiently the upper mediastinal lymph nodes. Recently, we reported that the left recurrent nerve runs at a different layer from the ramus cardiac us of the sympathetic nerve[13,14]. The communicating branch of these two nerves could be reserved. Complete dissection of the upper mediastinal lymph nodes, even if we usethe right thoracotomy approach, could be performed. In this report, we demonstrated that the dissection of the upper mediastinal lymph nodes could be performed via the left transcervical approach similarly to the right thoracotomy approach.We conclude that TCM-UMLD is a useful modality for the dissection of the upper mediastinal lymph nodesin cervical esophageal cancerbased on our experience with Thiel-embalmed human cadavers.

\section{References}

1. Thiel W (1992) The preservation of the whole corpse with natural color. Ann Anat 174(3): 185-195.

2. Thiel W (2002) Supplement to the conservation of an entire cadaver according to W Thiel. Ann Anat 184(3): 267-269.

3. Ida S, Morita M, Hiyoshi Y, Ikeda K, Ando K, et al. (2014) Surgical resection of hypopharynx and cervical esophageal cancer with a history of esophagectomy for thoracic esophageal cancer. Ann Surg Oncol 21(4): $1175-1181$

4. Grass GD, Cooper SL, Armeson K, Garrett-Mayer E, Sharma A (2014) Cervical esophageal cancer: A population-based study. Head Neck 37(6): 808-814.

5. Cao Z, Zheng J, Qian X, Sun Z (2014) Strategies of surgery treatment for cervical esophageal cancer. Zhonghua Wei Chang Wai Ke Za Zhi 17: $927-$ 930.

6. Tong DK, Law S, Kwong DL, Wei WI, Ng RW, et al. (2011) Current management of cervical esophageal cancer. World J Surg 35(3): 600-607.

7. Chou SH, Li HP, Lee JY, Huang MF, Lee CH, et al. (2010) Radical resection or chemoradiotherapy for cervical esophageal cancer? World J Surg 34(8): 1832-1839.

8. Hirano S, Nagahara K, Moritani S, Kitamura M, Takagita S (2007) Upper mediastinal node dissection for hypopharyngeal and cervical esophageal carcinomas. Ann Otol Rhinol Laryngol 116(4): 290-296.

9. Martins AS (2001) Neck and mediastinal node dissection in pharyngolaryngoesophageal tumors. Head Neck 23(9): 772-779.

10. Fujita H, Kakegawa T, Yamana H, Sueyoshi S, Hikita S, et al. (1999) Total esophagectomy versus proximal esophagectomy for esophageal cancer at the cervicothoracic junction. World J Surg 23: 486-491.

11. Okamoto M, Takahashi H, Yao K, Inagi K, Nakayama M, et al. (1995) Management of hypopharyngeal and cervical esophageal cancer (HCEC): a comparative study of primary-surgical and primary-radiotherapeutic regimens. Nihon Jibiinkoka Gakkai Kaiho 98(4): 571-578.

12. Jia SS, Nie SY, Chen YF, Liu FS, Li JD (1994) Surgical management of the hypopharyngeal and cervical esophageal cancer. Chin Med J (Engl) 107(12): 919-923.

13. Tokairin Y, Nagai K, Fujiwara H, Ogo T, Okuda M, et al. (2015) Mediastinoscopic subaortic and tracheobronchial lymph node dissection with a new cervico-hiatal crossover approach in thiel-embalmed cadavers. Int Surg 100(4): 580-588.

14. Tokairin Y, Nakajima Y, Kawada K, Hoshino A, Okada T, et al. (2018) Histological study of the thin membranous structure made of dense connective tissue around the esophagus in the upper mediastinum. Esophagus. 
ISSN: 2574-1241

DOI: 10.26717/BJSTR.2018.06.001362

Yutaka Tokairin. Biomed J Sci \& Tech Res

(c) This work is licensed under Creative

Submission Link: https://biomedres.us/submit-manuscript.php

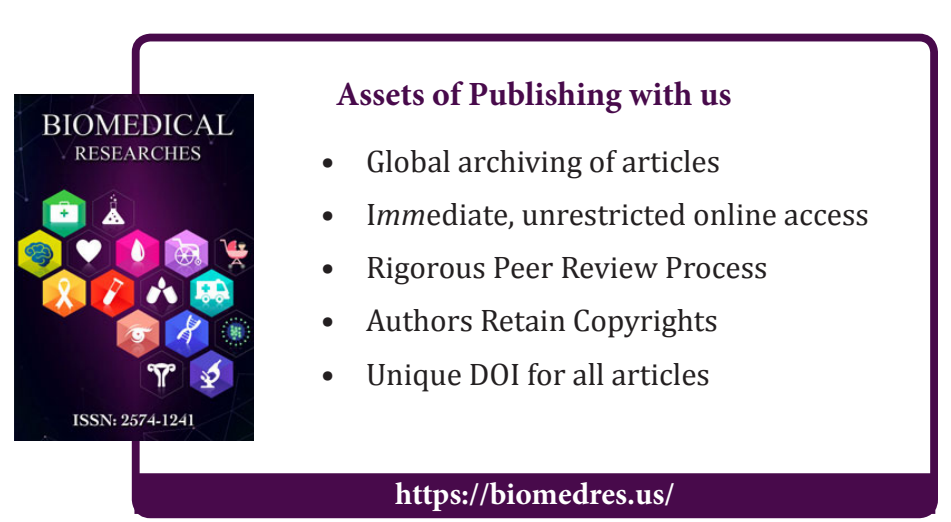

